\title{
Superconductivity Dome Rises from Damped Phonons
}

\author{
An unexpectedly simple extension to a theory explains the critical \\ temperature anomalies observed in ferroelectric superconductors. \\ By Rahul Rao
}

E ver since ferroelectric materials were first discovered, scientists have sought to understand how their properties relate to superconductivity. A recently discovered curiosity, for instance, is that these materials exhibit a puzzling "dome" in their critical superconducting temperature near their ferroelectric transitions. Now, Chandan Setty at Rice University, Texas, and colleagues provide a surprisingly simple explanation for this puzzle that uses lattice dynamics and phonons [1].

The superconducting dome is puzzling because of what the ferroelectric transition does to a material's phonons. According to Bardeen-Cooper-Schrieffer (BCS) theory, phonons mediate the binding of electrons into superconductivity-producing Cooper pairs. But as a material nears the ferroelectric transition-as a result of doping or the application of strain, for example-these phonons are damped and have their lifetimes reduced. In a conventional superconductor, such phonon damping lowers the critical superconducting temperature; in a ferroelectric material, the critical temperature instead rises to a

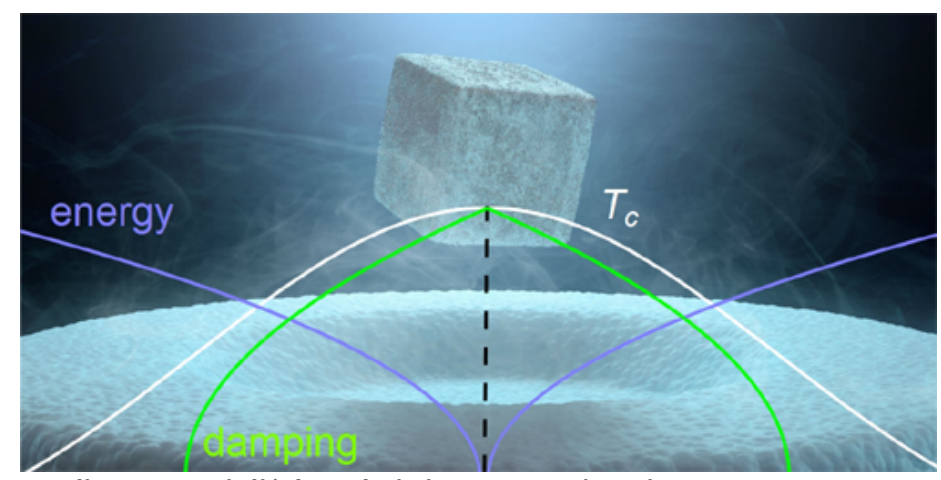

Credit: M. Baggioli/Shanghai Jiao Tong University;

ktsdesign/Shutterstock peak at the ferroelectric "instability point," where the damping is greatest.

Setty and colleagues explain the dome by expanding BCS theory so that it describes the phonon-damping process more accurately. In particular, they show that, at the instability point, this damping is anharmonic. As a result of this anharmonicity, anti-Stokes phonon-electron scattering is suppressed more than Stokes phonon-electron scattering. Since anti-Stokes scattering inhibits Cooper-pair formation, while Stokes scattering promotes it, the effect is an increase in the critical temperature around the instability point.

Anharmonic damping occurs in all ferroelectric materials-as well as other systems with so-called soft-mode structural instabilities-explaining why experimenters have observed superconducting domes across a wide range of conditions. Setty and colleagues hope that their new understanding will provide scientists with another tool to engineer superconductivity in such materials.

Rahul Rao is a freelance science writer based in New York.

\section{REFERENCES}

1. C. Setty et al., "Superconducting dome in ferroelectric-type materials from soft mode instability," Phys. Rev. B 105, L020506 (2022). 